\title{
GENERIC INTERACTION TECHNIQUES FOR MOBILE COLLABORATIVE MIXED SYSTEMS
}

\author{
P. Renevier ${ }^{*}$, L. Nigay ${ }^{*}$, J. Bouchet ${ }^{*}$, L. Pasqualetti ${ }^{\dagger}$ \\ *CLIPS-IMAG Laboratory, IIHM Team, University of Grenoble BP 53, 38041 Grenoble \\ Cedex 9, France \{Philippe.Renevier, Laurence.Nigay, Jullien.Bouchet\} @imag.fr \\ ${ }^{\dagger} F T$ R\&D-DIH/UCE 38-40 rue G. Leclerc 92794 Issy-lesMoulineaux, France \\ laurence.pasqualetti@francetelecom.fr \\ Abstract: The main characteristic of a mobile collaborative mixed system is that \\ augmentation of the physical environment of one user occurs through available \\ knowledge of where the user is and what the other users are doing. Links \\ between the physical and digital worlds are no longer static but dynamically \\ defined by users to create a collaborative augmented environment. In this \\ article we present generic interaction techniques for smoothly combining the \\ physical and digital worlds of a mobile user in the context of a collaborative \\ situation. We illustrate the generic nature of the techniques with two systems \\ that we developed: MAGIC for archaeological fieldwork and TROC a mobile \\ collaborative game.
}

Key words: Mixed Reality, Mobile Computing, CSCW, Interaction Techniques

\section{INTRODUCTION}

Mixed systems seek to smoothly link the physical and data processing environments. This is also the objective of other innovative interaction paradigms such as Ubiquitous Computing, Tangible Bits, Pervasive Computing and Traversable Interfaces. These examples of interaction paradigms are all based on the manipulation of objects of the physical environment [5]. Typically, objects are functionally limited but contextually relevant [8]. The challenge thus lies in the design and realisation of the fusion of the physical and data processing environments (hereafter called physical and digital worlds). The object of our study is to address this issue in the context of a collaborative mobility situation. Context detection and mixed 
reality are then combined in order to create a personalised augmented environment.

The structure of the paper is as follows: first, we define the context of our study by defining what mobile collaborative systems, mobile mixed systems and finally collaborative mixed systems are. We present related work and characterize existing systems highlighting the power and versatility of such systems. We then clarify the notion of mobile collaborative mixed systems. Having defined the goal and challenge of mobile collaborative mixed systems, we then present generic interaction techniques for smoothly combining the physical and digital worlds of a mobile user in the context of a collaborative situation. We illustrate the generic nature of the techniques with two systems that we developed: MAGIC for archaeological fieldwork and TROC a mobile collaborative game.

\section{RELATED WORK}

The objective of our study is to address the fusion of the physical and data processing environments in the context of a collaborative mobility situation. We therefore identify three intertwined ingredients: mobile systems, collaborative systems and mixed systems. In this section, we first study mobile collaborative systems, mobile mixed systems and then collaborative mixed systems.

\subsection{Mobile collaborative systems}

As computers become more and more prevalent, the need for systems that support coordination, communication and shared production between and within groups increases markedly. Such multi-user systems also called groupware have been made possible thanks to the advances of network technologies. A groupware may support synchronous interaction between users, such as a chat and/or asynchronous interaction between users such as email. In our study, we focus on mobile groupware. Mobile groupware are rapidly finding widespread use due to the recent progress in networking technologies. For example, a new protocol of continuous real time transport between a wireless network and a fixed network such as Ethernet is presented in [9]. This protocol is compatible with the quality of service of the current wireless networks. Moreover the studies carried out by the UMTS® consortium foresee, in the short run, flows of data of about 2Mbit/s. An example of existing collaborative systems is RAMSES [1], in the archaeology domain. Each archaeologist in the field takes notes on a Palmtop connected to a radio frequency (2 $\mathrm{Mb}$ a second) network so that notes can be shared by the 
group of archaeologists working in the same field.

Amongst mobile CSCW, the objective of our study is to understand the use of mobile supports and services required in a collaborative situation for a user's task in the real world. The aim is to create a seamless collaborative operational field between the physical and digital worlds, thanks to a socalled mixed system.

\subsection{Mobile mixed systems}

As we defined in [3], a mixed system is an interactive system combining physical and digital entities. Two classes of mixed systems are identified:

- Augmented Virtuality systems: Systems that make use of real objects to enhance the interaction between a user and a computer.

- Augmented Reality systems: Systems that enhance interaction between the user and her/his real environment by providing additional capabilities and/or information.

On the one hand, the Tangible User Interface paradigm [5] belongs to Augmented Virtuality: physical objects such as bricks are used to interact with a computer. On the other hand, the NaviCam system [10] and our Computer Assisted Surgery system CASPER [3] are two examples of Augmented Reality systems: the two systems display situation-sensitive information by superimposing messages and pictures on a video see-through screen (HMD, Head Mounted Display). The common design challenge of mixed systems (Augmented Virtuality as well as Augmented Reality systems) lies in the fluid and harmonious fusion of the physical and digital worlds.

In our study we focus on Augmented Reality systems, one class of mixed systems. The first Augmented Reality systems were designed for a specific use in a fixed environment. Progress made in wireless networks in terms of quality of services make it possible to build mobile Augmented Reality systems [6]. We believe that mobile Augmented Reality systems have a crucial role to play for mobile workers, bringing computer capabilities into the reality of the different workplaces. Systems already exist such as the Touring machine system of the project MARS (Mobile Augmented Reality Systems) [4] or the NaviCam system [10]. The user, while walking in a building such as a museum, in the streets or in a campus, obtains contextual information about the surrounding objects or about a predefined path to follow.

A mobile Augmented Reality system is one in which augmentation occurs through available knowledge of where the user is (the user's location and therefore the surrounding environment). Even though the user's location has an impact on the augmentation provided by the system, the latter does not 
necessarily maintain this location. Indeed, as explained in [6], on the one hand, the user's location and orientation are generally known by outdoor systems such as the Touring machine system, the position being tracked by a GPS. On the other hand, for indoor Augmented Reality systems, such as the NaviCam system, objects and places identify themselves to the system (RF, IR or video based tags): hence the system does not maintain the user's location.

To sum up, amongst mobile mixed systems, we focus on mobile Augmented Reality systems that enhance the interaction between the mobile user and her/his current real environment by providing additional capabilities and/or information.

\subsection{Collaborative mixed systems}

Several collaborative mixed systems have been developed. As for mobile mixed systems, we focus on one class of mixed systems, the Augmented Reality systems. As defined above, in Augmented Reality, interaction with the real world is augmented by the computer in order to assist a user in performing a task in the real world (i.e., modifying the real world). As a consequence, systems such as the StudierStub [13] that allows multiple collaborating users to simultaneously study three-dimensional scientific visualizations in a dedicated room is not part of our study because the task of studying a virtual object, is not in the real world. The shared real environment of the group of users is augmented by the computer but the task remains in the digital world.

An Augmented Reality system may provide support for shared production, communication and/or coordination amongst users. We call such systems Augmented Reality and Collaborative systems. We make a distinction between such Augmented Reality and Collaborative systems and the ones that we call Collaborative Augmented Reality systems that depict systems in which the physical environment of a group of users is collaboratively augmented. A Collaborative Augmented Reality system is one in which augmentation of the physical environment of one user occurs through the actions of other users. The main characteristic of a Collaborative Augmented Reality system is that augmentation of the physical environment of one user no longer relies on information pre-stored by the computer. Links between the physical and digital worlds are therefore dynamic, based on the users' actions and not defined in advance as for example in an augmented museum (the NaviCam system) [10]. Several Collaborative Augmented Reality systems exist and take on a variety of forms: In [11] we introduce a taxonomy of Collaborative Augmented Reality systems based on the classical distinction in 
groupware, that is the distance between users, as well as the distance between one or several users and the object of the task that belongs to the real world.

Amongst collaborative mixed systems, we focus on Collaborative Augmented Reality systems in which the physical environment of a group of users is collaboratively augmented.

\section{MOBILE COLLABORATIVE MIXED SYSTEMS}

Having introduced the context of our research, we now define what a mobile collaborative mixed system is. Such a system combines the characteristics of a mobile mixed system and of a collaborative mixed system. First a mobile mixed system, as defined above, is one in which augmentation occurs through available knowledge of where the user is (the user's location and therefore the surrounding environment). Second a collaborative mixed system is one in which augmentation of the physical environment of one user occurs through the actions of other users and no longer relies on information pre-stored by the computer. Links between the physical and digital worlds are therefore dynamic, based on the users' actions. Combining the characteristics of a mobile mixed system and of a collaborative mixed system, a mobile and collaborative mixed system is one in which augmentation occurs through available knowledge of where the user is and what the other users are doing.

Although mobile collaborative systems are now possible and systems already exist as explained in the previous section, and while some existing mixed systems are mobile and some are collaborative, few mixed systems combine the mobile and collaborative aspects. The main application domain of such systems is game and one of our developed system, TROC, is a game. Indeed, instead of recreating a virtual world, the existing games are based in the real world, the system only adding the magical possibilities related to the game rules. WARPING [12] is one example, but one of the users is not mobile, since s/he is in front of an augmented desktop. ARQuake [14] and Human-Pacman [2] are two additional examples of games. The users are mobile and they must kill digital enemies (ARQuake) or collect digital cookies (Human-Pacman). In these two examples, we can nevertheless notice that the links between the physical and digital worlds are predefined (positions of enemies or cookies) and the users can only destroy them, they cannot create new "links" such as putting a new cookie in the game field.

Beyond the HCI classical design approach, mobile collaborative mixed systems make it compulsory to use a multidisciplinary design approach that 
embeds complementary methods and techniques for the design and evaluation phases. In [7] we present a scenario-based design approach for mobile collaborative mixed systems. In particular scenarios enable the description of how the system would affect the way mobile users carry out their individual and collective activities. Based on the functions integrated in the so-called "projected scenarios", different interaction techniques can be designed. The interaction techniques, described in the following section, are generic and are those supported by our two mobile collaborative mixed systems: MAGIC dedicated to archaeological fieldwork and TROC a mobile collaborative game.

\subsection{Generic interaction techniques}

In order to explain the generic interaction techniques, we first describe the underlying hardware platform. This is an assembly of commercial pieces of hardware. The platform includes a Fujitsu Stylistic pen computer. This pen computer runs under the Windows operating system, with a Pentium III (450 $\mathrm{MHz}$ ) and $196 \mathrm{Mb}$ of RAM. The resolution of the tactile screen is $1024 \times 768$ pixels. In order to establish remote mobile connections, a WaveLan network by Lucent (11 Mb/s) was added. Connections from the pen computer are possible at about 200 feet around the network base. The hardware platform also contains a Head-Mounted Display (HMD), a SONY LDI D100 BE: its semi-transparency enables the fusion of computer data (opaque pixels) with the real environment (visible via transparent pixels). Secondly, a (D-)GPS is used to locate the users. Finally, capture of the real environment by the computer is achieved by the coupling of a camera and an orientation sensor. We first used an absolute orientation sensor, the magnetometer HMR3000 by Honeywell. We now use an intertrax 2 that is more accurate. The camera orientation is therefore known by the system. Indeed the orientation sensor and the camera are fixed on the HMD, in between the eyes of the user. The system is then able to know the position (GPS) and orientation (magnetometer or intertrax) of both the user and the camera. Figure 1 shows a user, fully equipped: the equipment is quite invasive and suffers from a lack of power autonomy. Our goal is to demonstrate the feasibility of our interaction techniques by assembling existing commercial pieces of hardware and not by designing specific hardware out of the context of our expertise. For a real and long use of the platform in a "real" site, a dedicated hardware platform must clearly be designed. 


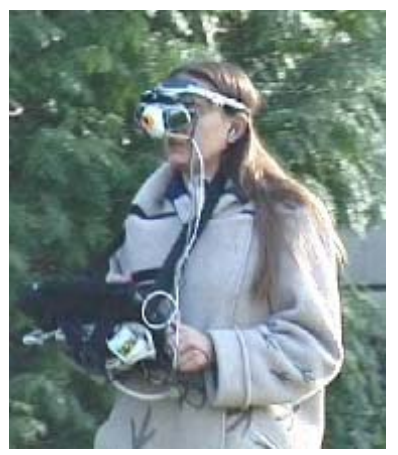

Figure 1. A user wearing and holding the hardware platform

The mobile users manipulate objects that are either digital or physical. Interaction techniques must be designed in order to let them manipulate the two types of objects: physical and digital. For flexibility and fluidity of interaction, such manipulation is either in the physical world or in the digital world. We therefore obtain four cases, by combining the two types of objects and the two worlds: the physical world (i.e., the archaeological field or the game ground) and the digital world (i.e., the screen of the pen computer):

1. Interaction with a physical object in the digital world: Mixed interaction.

2. Interaction with a digital object in the physical world: Mixed interaction.

3. Interaction with a physical object in the physical world: Interaction purely in the real world.

4. Interaction with a digital object in the digital world: Interaction in the digital world (graphical user interface).

In [7] we fully describe the four types of interaction. We focus here on the interaction techniques corresponding to the types (1) and (2). For both cases, passive and active interaction techniques are designed. Passive interaction techniques are based on tracking mechanisms (such as localization and orientation of the mobile user). With passive techniques, the user does not explicitly issue a command to the system as opposed to active interaction techniques that correspond to the case where the user issues a command to the system, for example a drag\&drop of an object.

The two types of mixed interaction ((1) and (2)) respectively imply (i) that physical objects must be manageable in the digital world (ii) that digital objects must be manageable in the physical world. To do so we designed a generic interaction technique, a gateway that plays the role of a door between the physical and digital worlds. As a door belongs to two rooms, the gateway exists in both worlds:

- the gateway is an area of the physical world, delimited by a rectangle displayed in a semi-transparency Head-Mounted Display (HMD) as 
shown in Figure $2 b$,

- the gateway is a rectangular area in the digital world, on the pen computer screen as shown in Figure 2a (window entitled "Head Mounted Display").

Concretely the gateway is simply a window both displayed on the HMD (Java JFrame) on top of the physical world and on the pen computer screen (Java JInternalFrame). As opposed to the Touring Machine system [4] in which the pen computer is used to display information about the surrounding physical environment of the user that is not displayed in the HMD, objects in the gateway are visible on the HMD (i.e., in the physical world) as well as on the pen computer screen (i.e., in the digital world), as shown in Figure 2.

Based on the gateway, we designed two interaction techniques, namely the "clickable reality" and the "augmented field".

- The "Clickable reality" technique: from the physical world to the digital world. If the object is physical (1), the object is transferred to the digital world thanks to the camera (fixed on the HMD, between the two eyes of the user). The real environment captured by the camera is displayed in the gateway window on the pen computer screen as a background. We allow the user to select or click on physical objects: we therefore call this technique "the clickable reality". Before taking a picture, the camera must be calibrated according to the user's visual field. Using the stylus on screen, the user then specifies a rectangular zone thanks to a magic lens (a type of camera lens). The cursor displayed on the pen computer screen is also displayed on top of the physical world. The corresponding specified zone (magic lens), displayed in the gateway window on screen and on the HMD, corresponds to the physical object to be captured. The picture can then be stored in the shared database along with the location of the object. Note that although the user is manipulating a magic lens using the stylus on screen, s/he perceives the results of her/his actions in the physical world.

- The "Augmented field" technique: from the digital world to the physical world. If the object is digital (2) dragging it inside the gateway makes it visible in the real world. For example the user can drag a drawing or a picture stored in a database to the gateway window. The picture will automatically be displayed on the HMD on top of the physical world as shown in Figure $2 \mathrm{~b}$. Moving the picture using the stylus on the screen will move the picture on top of the physical world. This action is for example used if a user wants to compare an object from a database with a physical object in the field. Putting them next to each other in the real world will help their comparison. The motion of a digital object (ex: drag and drop on the pen computer) can be viewed by the user without looking at the pen computer screen. This is because in using the 
HMD the user can simultaneously view digital objects and the real world. Although the user is manipulating a digital object, s/he perceives the results of her/his actions in the physical world.

First, transfer of digital objects to the physical world can be explicitly managed by the user by drag and drop (active interaction technique) as explained above or can be automatic (passive interaction technique). Automatic transfer is performed by the system based on the current location of the user.

Second, transfer of digital objects to the physical world can be transient or persistent. Indeed, on the one hand, transfer of digital objects to the physical world can be transient as for comparing a digital object from a database with a physical found object. On the other hand, transfer of digital objects to the physical world performed by one user can be persistent so that later on other users can discover such digital objects that augment the physical environment. Such a technique is called "augmented field". When a user walks in the site, s/he can see discovered objects specified by colleagues. The "augmented field" is an example of asynchronous collaboration. It is therefore a generic technique for mobile collaborative Augmented Reality system.

These generic interaction techniques (i.e., the "gateway" technique on which the "clickable reality" as well as the "augmented field" techniques rely) are supported by two mobile collaborative mixed systems that we developed: MAGIC dedicated to archaeological fieldwork and TROC a mobile collaborative game.

\section{SYSTEMS: MAGIC AND TROC}

\subsection{MAGIC for archaeological fieldwork}

The design of the MAGIC system is based on a study of the tasks of archaeological fieldwork, interviews and observations in Alexandria (Egypt) [7]. The archaeological fieldwork in Alexandria is time-constrained because the archaeological site must be explored in less than three months (rescue archaeology). Tools that can make such fieldwork more efficient are therefore important. This is a suitable application domain for mobile collaborative mixed systems because archaeologists work in groups, moving in a delimited site and requiring collections of data. Figure 2a presents the graphical user interface of MAGIC on the pen computer. Coordination between users relies on the map of the archaeological site, displayed within a dedicated window (at the bottom left corner of Figure 2a). For each found object, archaeologists 
fill a form describing the object, draw some sketches or very precise drawings and take pictures using the "clickable reality" technique. Analysis of objects relies on comparisons with known objects ("Augmented field” technique) from other archaeologists or reference manuals (database) and on discussions with other archaeologists in the site or with a distant expert. Figure 2b, a reconstituted picture, presents a such comparison. After validation, the object is then added to the shared database and is visible on the map of each user. Because a picture is stored along with the location of the object, we can restore the picture in its original real context (2D representation). When an archaeologist walks in the site, s/he can see discovered objects removed from the site and specified in the database by colleagues ("Augmented field" technique). S/he can then see the object as it was before being removed from the site. The "augmented field" technique is particularly useful to see objects belonging to a stratum higher than the current one, because by definition the objects have all been removed. The MAGIC system along with its software architecture is fully described in [11]. Although the design is based on task and activity analysis performed in Alexandria (Egypt), we were not able to experimentally test MAGIC on a site there. In order to show the generic aspect of our techniques and also to be able to perform experimental tests we developed a second application, TROC, a collaborative game.

\subsection{TROC: a mobile collaborative game}

TROC (barter in French) is a mobile collaborative game. Each player has to collect a list of digital objects that are positioned in the game field at the beginning of the game. As shown in part B of Figure 3, the digital objects to be collected are animals (cat, gull, etc.). Thanks to the "augmented field" technique, the player while moving discovers the objects. TROC also includes 3D sounds that help the player to find the objects. In addition the player can use "magical tools" to locate the objects as well as the other players on the map displayed on the pen computer (part D of Figure 3, the round circle specifying the zone of observation). The player can also specify filters (part A of Figure 3) so that s/he will only see one kind of digital object, in the physical world (the game field) as well as on the map. Digital objects collected by a user are stored in four physical cubes carried by the player. The content of the four cubes is displayed on the pen computer (part $\mathrm{C}$ of Figure 3) as well as on top of the physical cube recognized by a vision algorithm thanks to the camera fixed on the HMD. To collect a digital object, the player has two possibilities: first s/he can use the "clickable reality" technique or s/he can present a physical cube to the camera fixed on the HMD while issuing the voice command "take". The player can also empty a cube and put back on the game field a previously collected digital object ("augmented 
field" technique). This is an example of asynchronous collaboration between players. In order to win and collect her/his assigned list of objects, the players must collaborate and exchange collected objects. The game is based on the barter technique. During exchanges, a player can lie saying that s/he has a given object and can also give a trapped object to another player.

a)

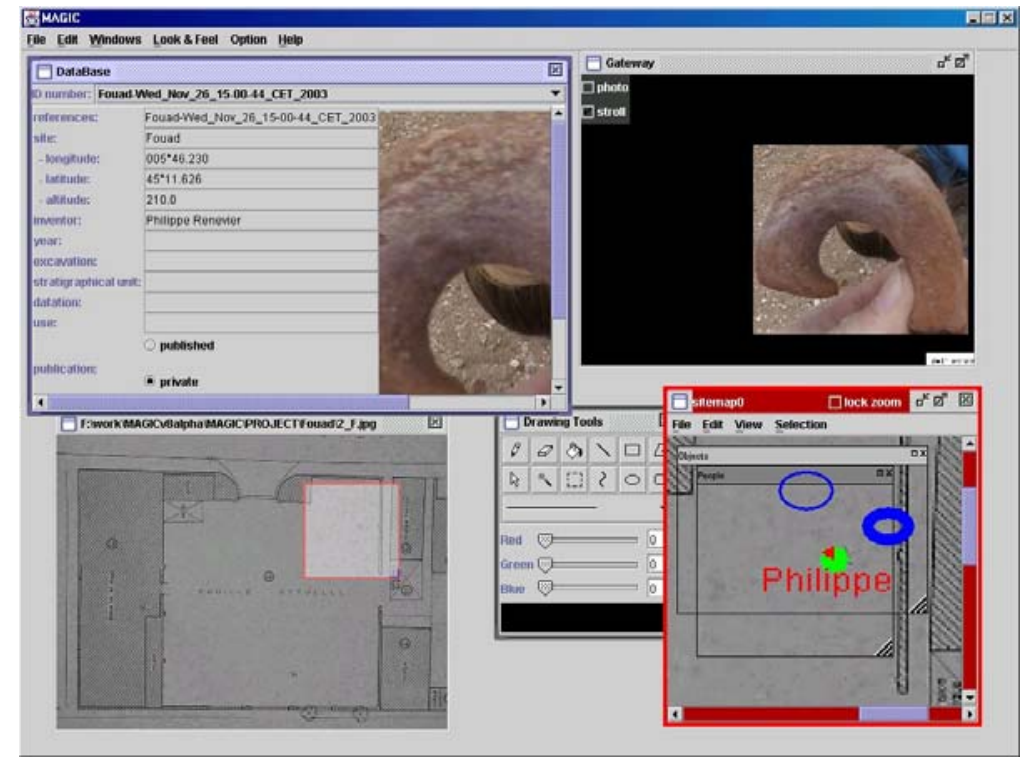

b)

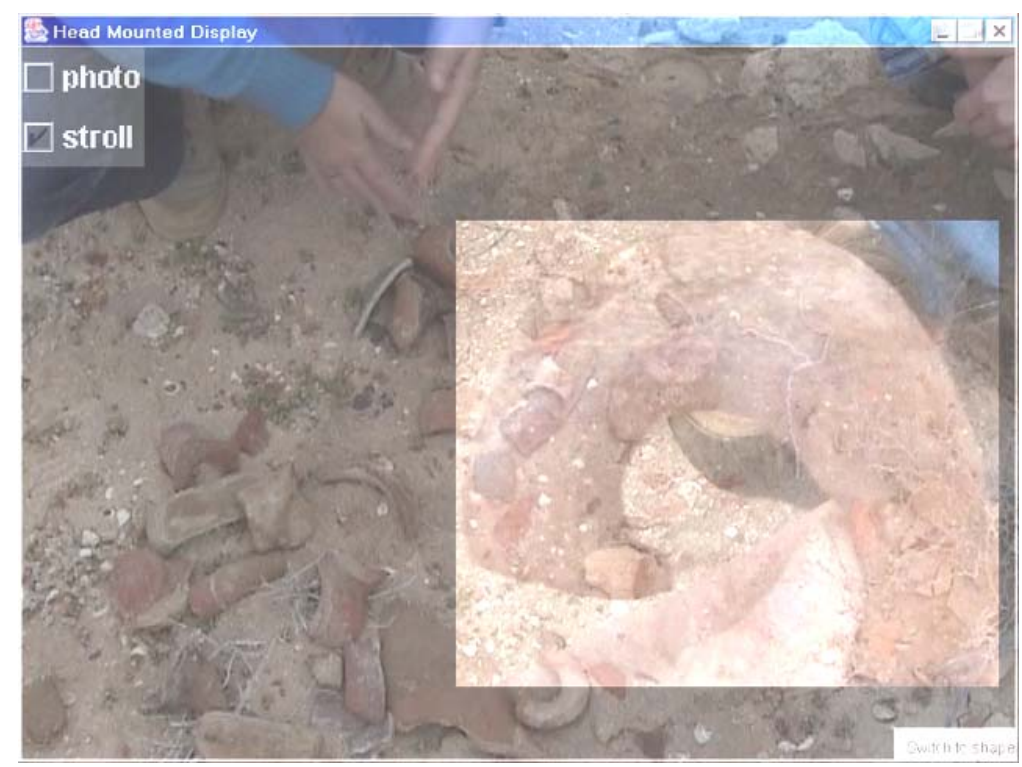

Figure 2. MAGIC system (a) User interface on the pen computer (b) View displayed on the HMD 


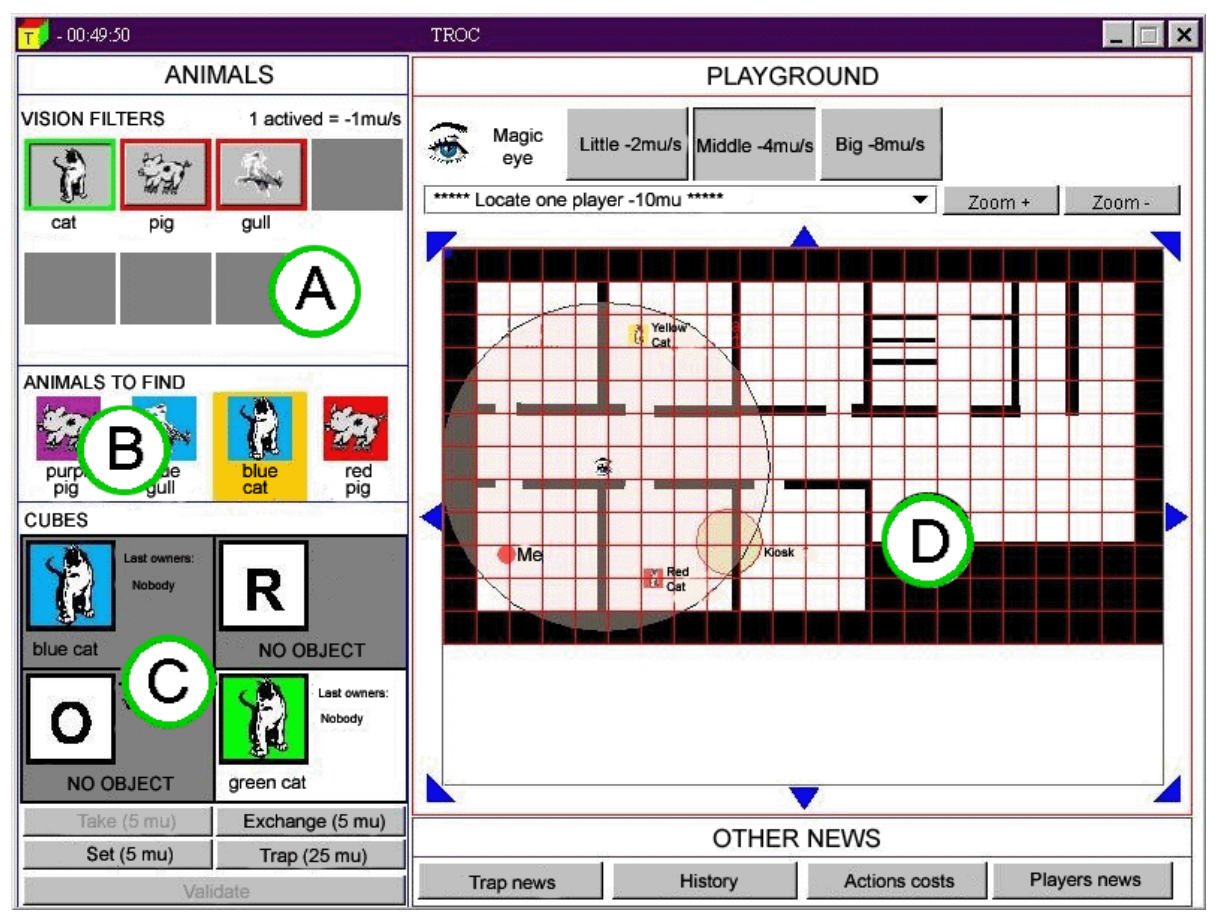

Figure 3. User interface of TROC

We performed a first set of experimental tests of TROC. Two functions were simulated (wizard of oz technique): the voice recognition and the location of a player. We had one wizard per player. In addition, during this first set of tests, the players did not have to manipulate the physical cubes. The objective was to study the impact of the physical environment (with or without landmarks) and of 3D sound on localizing the digital objects. Eight volunteers all familiar with computers participated in the experiment. A first phase enables the players to get familiar with the rules of the game and the techniques. Then four experimental settings were studied. (1) without 3D sound (2) with 3D sound (3) in a game field without physical landmark, (a big empty room) (4) in a game field with physical landmarks, (with rooms and a corridor). Four players played the game twice in the two following experimental settings: first, without 3D sound and then with 3D sound in a field without physical landmark. The four other players also played the game twice in the two following experimental settings: first with 3D sound and then without 3D sound in a game field with physical landmarks. So each player played four games. For each game, the test was finished when a player collected all the objects assigned to her/him. After each game, interviews were conducted with the players.

The primary analysis of the collected data shows that 3D sounds facilitate 
the location of digital objects, sound being available before the object is visible. In addition, the players underlined the fact that the sound reinforces the link between the physical and digital worlds, by making digital objects more real. In addition, it has been observed that digital objects, the focus of the players, had a strong presence to the point that players forgot the physical obstacles. Players underlined the inconsistency of seeing an object through a wall and having to go inside the room to be able to pick it up. Although such a possibility was presented as a magical tool which allows one to see through the walls, it confirms the fact that consistency must be maintained while combining the physical and digital worlds. The participants also wanted to pick up objects by hand. In particular such behavior has been observed when the objects were very close to the players and therefore very big. Moreover players had more difficulties to locate objects in a game field without physical landmarks. Indeed, they adopted an approach of blind searching, while with physical landmarks they first located the objects on the map and then went to pick them up.

\section{FUTURE WORK}

The generic techniques, "gateway", "clickable reality” and "augmented field", define a reusable hardware and software platform. As on-going work, we are pursuing two avenues.

First we are currently reusing and extending the platform for new applications: we are developing a system that allows users to annotate physical locations with digital notes, which are then read/remove by other mobile users. The presented interaction techniques therefore constitute the first bricks of a toolkit for developing mobile collaborative mixed systems. Reusability of the code and independence of part of it with the hardware are guaranteed by the software architecture model that we applied for developing the platform [11].

Our second research avenue is experimental. Further experimental tests will be performed with the TROC game and the new applications developed with the platform. Our objective is to gain understanding of how the users perceive and interact within the combined physical/digital world. For example, we plan to study when the player selects interaction techniques in the physical world as opposed to interaction techniques in the digital world. To do so, functionally equivalent interaction techniques such as manipulation of physical cubes and direct manipulation on the pen computer are provided. 


\section{ACKNOWLEDGEMENTS}

This work is supported by France Telecom R\&D, under contract Houria $\mathrm{N}^{0}$ AJC067CH. Special thanks to G. Serghiou for reviewing the paper.

\section{REFERENCES}

[1] M. Ancona, G. Dodero, V Gianuzzi, "RAMSES: A Mobile Computing System for Field Archaeology”, Handheld and Ubiquitious Computing 1st Int'l Symp. (HUC’99), Karlsruhe, Springer-Verlag, LNCS 1707, 1999, pp 222-233.

[2] A. Cheok, S. Fong, K. Goh, X. Yang, W. Liu, F. Farbiz, Y. Li, "Human Pacman: A Mobile Entertainment System with Ubiquitous Computing and Tangible Interaction over a Wide Outdoor Area”, Proc. mobile HCI 2003, Springer Verlag LNCS 2795, Udine, September 2003, pp 209-223

[3] E. Dubois, L. Nigay, J. Troccaz, O. Chavanon, L. Carrat, "Classification Space for Augmented Surgery, an Augmented Reality Case Study”, Proc. 7th Conf. HumanComputer Interaction (Interact'99), ACM, Edinburgh, 1999, pp 353-359.

[4] S. Feiner, B. MacIntyre, T. Hollerer, A. Webster, “A Touring Machine: Prototyping 3D Mobile Augmented Reality Systems for Exploring the Urban Environment”, Proc. 1st Int'l Symp. Wearable Computers (ISCW 97), IEEE CS Press, Los Alamitos, 1981, pp. 439-449.

[5] H. Ishii, B. Ullmer, “Tangible Bits: Towards Seamless Interfaces between People, Bits and Atoms”, Proc. Conf. Human Factors in Computing Systems (CHI '97), ACM, Atlanta, March 1997, pp. 234-241.

[6] K. Kangas, J. Röning, "Using Code Mobility to Create Ubiquitous and Active Augmented Reality in Mobile Computing”, Proc. Int'l Conf. Mobile Computing and Networking (Mobicom' 99), ACM, Seattle, 1999, pp 48-58.

[7] L. Nigay, P. Salembier, T. Marchand, P. Renevier, L. Pasqualetti, "Mobile and Collaborative Augmented reality: A Scenario Based Design Approach”, Proc. Mobile HCI 2002, Springer-Verlag, LNCS 2411, Pisa, 2002, pp. 241-255.

[8] D. Norman, “The design of everyday things”, Basic Books, September 2002.

[9] T. Pyssyalo, T. Repo, T. Turunen, T. Lankila, J.Röning, "CyPhone - Bringing Augmented Reality to Next Generation Mobile Phones”, Proc. Designing Augmented Reality Environments (DARE’2000), ACM, Elsinore, 2000, pp. 11-21.

[10] J. Rekimoto, “Navicam: A Magnifying Glass Approach to Augmented Reality”, Presence: Teleoperators and Virtual Environments, Vol. 6, No. 4, MIT Press, 1997, pp.399-412.

[11] P. Renevier, L. Nigay, "Mobile Collaborative Augmented Reality: the Augmented Stroll”, Proc. Engineering for Human Computer Interaction (EHCI'01), Springer-Verlag, LNCS 2411, Toronto, 2001, pp. 315-334.

[12] T. Starner, B. Leibe, B. Singletary, J. Pair, "MIND-WARPING: Towards Creating a Compelling Collaborative Augmented Reality Game”, International Conference on Intelligent User Interfaces (IUI 2000), New Orleans, ACM, 2000, pp. 256-259.

[13] Z. Szalavári, D. Schmalstieg, A. Fuhrmann, M. Gervautz, "Studierstube An Environment for Collaboration in Augmented Reality”, Journal of the Virtual Reality Society "Virtual Reality : Research, Development and Application”, Virtual Press Ltd., UK, 1997.

[14] B. Thomas, B. Close, J. Donoghue, J. Squires, P. De Bondi, W. Piekarski, "First Person Indoor/Outdoor Augmented Reality Application: ARQuake”, Personal and Ubiquitous Computing, Vol. 6 No. 1, Electronic Edition (Springer LINK), 2002, pp. 75-86. 